\title{
Quantify osteoarthritis gait at the doctor's office: a simple pelvis accelerometer based method independent from footwear and aging
}

\author{
R. Barrois ${ }^{a}$, L. Oudre ${ }^{a, e}$, Th. Moreau a,e, Ch. Truong ${ }^{a, e}$, N. Vayatis ${ }^{a, e}$, S. Buffat ${ }^{a}$, A. Yelnik ${ }^{a}$, C. de Waele ${ }^{a}$, Th. Gregory ${ }^{b}$, \\ S. Laporte ${ }^{c}$, P. P. Vidal ${ }^{a}$ and D. Ricard ${ }^{a, d}$ \\ ${ }^{a}$ Cognition and Action Group, Cognac-G, CNRS, Université Paris Descartes, SSA, Paris, France; 'bervice de chirurgie orthopédique et de \\ traumatologie de l'Hôpital Européen Georges Pompidou, Paris, France; 'LBM/Institut de Biomécanique Humaine Georges Charpak, Arts et \\ Métiers Paris Tech, Paris, France; 'Service de Neurologie de l'Hôpital d'Instruction des Armées du Val de Grâce, Service de Santé des Armées, \\ Paris, France; ${ }^{e} \mathrm{CMLA}$ - ENS Cachan, Cachan, France
}

KEYWORDS Gait analysis; doctor's office; osteoarthritis

\section{Introduction}

The gold standard to evaluate the severity of osteoarthritis in the doctor's office remains clinical scores (Bellamy 2002). The Western Ontario and McMaster Universities (WOMAC) osteoarthritis index is the most largely used score in rheumatology for lower limb osteoarthritis. It is based on clinical observation and it assesses pain, stiffness, and physical function in patients with hip and knee osteoarthritis. It is valid, reliable, and sensitive to evaluate osteoarthritis and adapted to doctor's office (Bellamy 2002).

However, clinical scores are inherently subjective and they depend from the patient's impression and from the clinician's interpretation. Gait analysis in modern gait laboratories with force plates and photogrammetry is a good tool to have an objective, quantified, and precise insight in osteoarthritis (Astephen et al. 2008).

For practical reasons, skin-mounted inertial sensors are well suited for investigating gait kinematics (Auvinet et al. 2002). In accelerometer-based gait analysis, aging is also known to affect gait parameters (Oberg et al. 1993). To have a clinical measure of osteoarthritis, it is essential to find a technique that is independent from aging. Footwear can also affect walking parameters (Chambon et al. 2014). Since it is too time consuming to ask the patient to take off his shoe for the measurement, it is essential to find a method independent from the shoe type.

Walking ten meters go and ten meters back on a level surface at comfortable walking speed is a well-suited protocol for clinical situations.

This study proposes to test a $3 \mathrm{D}$ pelvis accelerometer-based measurement method on a group of 47 patients suffering from lower limb osteoarthritis and 12 asymptomatic subjects. The aim was to see whether the accelerometer-based method is correlated with the clinical severity of the lower limb osteoarthritis evaluated with the WOMAC index. In addition, this study evaluates whether the accelerometer-based method is independent of aging on 75 asymptomatic subjects and whether the accelerometer-based method is independent from footwear on one asymptomatic subject.

\section{Methods}

\subsection{Subjects}

Forty-seven osteoarthritis patients had hip or knee osteoarthritis diagnosed and graded with the WOMAC index (0-96, 43-90 years, mean 70.9 years) by the same surgeon. Twelve asymptomatic subjects were included in the study (40-87 years, mean 60.8 years). Participants were divided into four severity groups. The repartition was based on the WOMAC index. Score ranges were fixed in order to obtain three groups of same score amplitude.

- Severity group 0: asymptomatic subjects (from 40 to 87 years).

- Severity group 1: WOMAC from 1 to 32 (slightly impaired subjects).

- Severity group 2: WOMAC from 33 to 64.

- Severity group 3: WOMAC from 65 to 96 (severely impaired subjects).

To assess aging as a confounding factor, 75 additional asymptomatic subjects were included in the study (18-40 years, 27.3). Asymptomatic participants were divided into four age groups.

- Age group 0: age from 18 to 39 .

- Age group 1: age from 40 to 59 .

- Age group 2: age from 60 to 79.

- Age group 3: age more than 80.

To assess footwear as a confounding factor, one subject of 25 years old was asked to perform the walking task in different footwear conditions.

Both patients and asymptomatic subjects gave their written consent to participate in this study. 

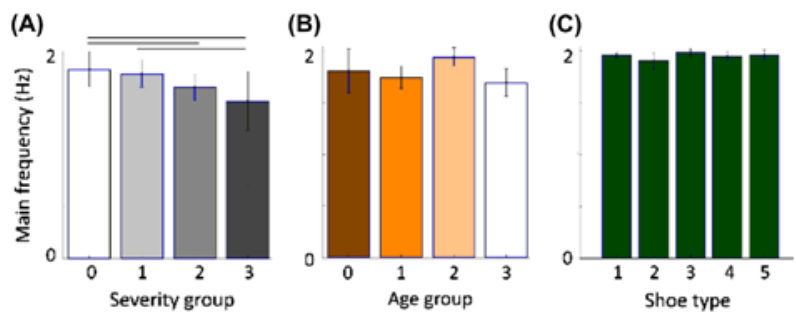

Figure 1. (A) Main frequency as a function of WOMAC index-based severity groups built with 12 asymptomatic and 47 symptomatic subjects. Mean values (gray bars) and standard deviations are shown for each group. ANOVA analysis with Tukey's pairwise comparison test was performed. Horizontal black lines indicate statistically significant differences ( $p$-value $<0.05$ ). (B) Main frequency as a function of age groups built with 75 asymptomatic subjects. (C) Main walking frequency for the same asymptomatic participant walking with five different shoe types.

\subsection{Instrumentation}

Pelvis linear acceleration was collected using one triaxial accelerometer (XSens ${ }^{\oplus}$ MTw Measurement Units $100 \mathrm{~Hz}$ ). The sensor was fixed on the pelvis (L4-L5 vertebra) using manufacturerdesigned adhesive Velcro ${ }^{\circledR}$ tape.

\subsection{Experimental design and data acquisition}

First, WOMAC index was evaluated and recorded by the same orthopedic surgeon. After the sensor fixation, the participant was instructed to execute the following sequence: stand quiet for $6 \mathrm{~s}$, walk $10 \mathrm{~m}$ at preferred walking speed on a level surface, make a $\mathrm{U}$ turn, walk back at preferred walking speed on a level surface, stand quiet for $2 \mathrm{~s}$.

Participants could keep their clothes and their shoes on. Each participant made two runs of this exercise to improve the reliability of the measure.

To assess footwear as a confounding factor, the same subject walked five runs in five different footwear conditions ( 25 trials). The shoe types were as follows: Type 1: boots; Type 2: running; Type 3: classical; Type 4: socks; Type 5: sneakers.

\subsection{Experimental design and data acquisition}

The phases of the exercise (quiet standing, walking, and U turn) were manually annotated. Main frequency given by the major peak of the Fast Fourier Transform was computed on the manually annotated walking phases of the exercise including initiation of gait and gait termination. The mean value of the result on the two runs was taken. For the influence of shoe experiment, the mean value on the five runs was taken.

\subsection{Statistical analysis}

ANOVA analysis with Tukey's pairwise comparison test was performed.

\section{Results and discussion}

We observed a decrease of the walking main frequency with the severity of osteoarthritis (Figure $1(\mathrm{~A})$ ). Differences were significant for the following pairs: $0-2 ; 0-3 ; 1-3$. No clear correlation was found between main walking frequency and aging (Figure 1(B)) and between walking frequency and the footwear conditions (Figure 1(C)).

The objective of our method was to setup a pelvis accelerometer-based method adapted to the doctor's office that has the potential to be correlated with the clinical WOMAC index-assessed osteoarthritis severity in 47 patients and 12 asymptomatic subjects walking go and back ten meters in clinical consultation conditions. This method is meant to be independent from footwear and aging.

In our study, there is a difference in age between the 12 asymptomatic subjects (mean 60.8 years) and the 47 symptomatic subjects (mean 70.9 years). The walking frequency is known to decrease from about $0.05 \mathrm{~Hz}$ between 60 and 70 years in asymptomatic subjects (Oberg et al. 1993). The differences observed with increasing severity are around $0.15 \mathrm{~Hz}$ in this study.

This study shows a decrease in walking frequency with increasing lower limb osteoarthritis severity but not with aging nor with the change of footwear condition. This makes main walking frequency an interesting simple parameter to assess osteoarthritis severity with pelvic acceleration.

\section{Conclusions}

This pelvis accelerometer-based method shows a trend correlation with the WOMAC index. This method seems independent from aging and footwear and is well suited for daily use at the doctor's office.

\section{References}

Astephen JL, Deluzio kJ, Caldwell GE, Dunbar MJ, Hubley-Kozey CL. 2008. Gait and neuromuscular pattern changes are associated with differences in knee osteoarthritis severity levels. J Biomech. 41:868-876.

Auvinet B, Berrut G, Touzard C, Moutel L, Collet N, Chaleil D, Barrey E. 2002. Reference data for normal subjects obtained with an accelerometric device. Gait Posture. 16:124-134.

Bellamy N. 2002. WOMAC: a 20-year experimental review of a patient-centered self-reported health status questionnaire. J Rheumatol. 29:2473-2476.

Chambon N, Delattre N, Guéguen N, Berton E, Rao G. 2014. Is midsole thickness a key parameter for the running pattern? Gait Posture. 40:58-63.

Oberg T, Karsznia A, Oberg K. 1993. Basic gait parameters: reference data for normal subjects, 10-79 years of age. J Rehabil Res. 30:210-223. 